\title{
Bi-scalar modified gravity and cosmology with conformal invariance
}

\author{
Emmanuel N. Saridakis ${ }^{a, b}$ Minas Tsoukalas $^{c}$ \\ ${ }^{a}$ CASPER, Physics Department, Baylor University, Waco, TX 76798-7310, USA \\ ${ }^{b}$ Instituto de Física, Pontificia Universidad de Católica de Valparaíso, Casilla 4950, Valparaíso, \\ Chile \\ ${ }^{c}$ Physics Department, Boğaziçi University, 34342, Bebek, Istanbul, Turkey \\ E-mail: Emmanuel_Saridakis@baylor.edu, minasts@central.ntua.gr
}

\begin{abstract}
We investigate the cosmological applications of a bi-scalar modified gravity that exhibits partial conformal invariance, which could become full conformal invariance in the absence of the usual Einstein-Hilbert term and introducing additionally either the Weyl derivative or properly rescaled fields. Such a theory is constructed by considering the action of a non-minimally conformally-coupled scalar field, and adding a second scalar allowing for a nonminimal derivative coupling with the Einstein tensor and the energy-momentum tensor of the first field. At a cosmological framework we obtain an effective dark-energy sector constituted from both scalars. In the absence of an explicit matter sector we extract analytical solutions, which for some parameter regions correspond to an effective matter era and/or to an effective radiation era, thus the two scalars give rise to "mimetic dark matter" or to "dark radiation" respectively. In the case where an explicit matter sector is included we obtain a cosmological evolution in agreement with observations, that is a transition from matter to dark energy era, with the onset of cosmic acceleration. Furthermore, for particular parameter regions, the effective dark-energy equation of state can transit to the phantom regime at late times. These behaviors reveal the capabilities of the theory, since they arise purely from the novel, bi-scalar construction and the involved couplings between the two fields.
\end{abstract}

KEYwords: Bi-scalar theories, Modified gravity, Conformal invariance, Dark energy, Inflation 


\section{Contents}

1 Introduction 1

2 Action and field equations 3

3 Cosmology 5

$\begin{array}{lll}4 & \text { Solutions } & 7\end{array}$

$\begin{array}{lll}4.1 & \text { Case 1: } c_{0}=0 & 7\end{array}$

4.2 Case 2: $16 \pi G \eta \beta-\gamma=0 \quad 9$

$\begin{array}{llr}4.3 \text { General case } & 10\end{array}$

$\begin{array}{lll}4.4 & \text { Numerical elaboration } & 12\end{array}$

5 Conclusions $\quad 14$

\section{Introduction}

The motivation for a gravitational modification, i.e the construction of a modified theory of gravity that possesses General Relativity as a particular limit is twofold. On one hand, from purely theoretical considerations, such a modification could improve the renormalizability issues of General Relativity, possibly opening the way towards gravitational quantization [1]. On the other hand, one has the cosmological motivation, namely the hope that a gravitational modification could describe the observed late-time universe acceleration and/or the early-time inflationary era [2], without the need of dark energy [3, 4] or of the inflaton field [5].

One of the main requirements when modifying gravity is the preservation of secondorder field equations, which protects the theory from ghost instabilities [6-8]. A nice paradigm along this direction are the scalar-tensor theories, which provide one of the simplest ways to deviate from General Relativity, and have long been extensively investigated [9]. Recently, they have regained a significant amount of attention, with the resurrection $[10,11]$ and rediscovery [12] of the most general scalar tensor theory in four dimensions, with second-order field equations for the metric and the scalar field, presented originally in the 70's by Horndeski [13]. Additionally, lately there have also been attempts to go beyond Horndeski's original theory, in terms of the allowed number of derivatives in the field equations, and allow for higher derivative theories which nevertheless can be cast into second-order form [14-17]. In these theories the degrees of freedom remain $2+1$ regardless of the chosen gauge [18-22], and the existence of a specific primary constraint allows for such theories to survive [23].

Following the above lines, one could try to modify gravity by adding more extra degrees of freedom to General Relativity, expressing them as additional scalars, resulting to 
bi-scalar gravitational theories, possessing $2+2$ ghost-free degrees of freedom. Such biscalar theories were originally formulated through extensions of Galileon theories [24-26], including one extra scalar field [27]. It was later conjectured that the most general covariant multi-scalar-tensor theory arises as a natural generalization of Horndeski's theory [28], nevertheless it was shown that the proposed theory is actually the most general in a flat background, but not in any curved geometry [29, 30]. The most general second-order field equations for a bi-scalar theory where recently presented in [31], while in [32] the authors constructed the Jordan-frame version of general classes of bi-scalar theories, which prove to have interesting cosmological implications [33]. Moreover, bi-scalar theories can have interesting phenomenology [34]. Finally, examining the behavior of these theories under conformal transformations could help to reveal their underlying properties [35].

On the other hand, it is know that conformal invariance is an important property of a theory, both theoretically as well as concerning its applications. In particular, a gravitational modification with full or partial conformal invariance has the theoretical interest that it could be a useful tool towards the exploration of physics close to the Planck scale [36]. Additionally, it can have important cosmological implications since it can naturally lead to (almost) scale invariant spectrum of primordial density fluctuations, in agreement with observations [37, 38]. Moreover, scale/conformal invariance appears to be an important ingredient in early universe cosmology [39-44], giving rise to a wide range of inflationary models [45]. Furthermore, it can also lead to interesting black hole physics [46]. Finally, there have been numerous attempts to explore conformal invariance through Weyl gravity $[47,48]$ and its connection with General Relativity [49].

A bi-scalar theory exhibiting partial conformal invariance was constructed in [50], which can exhibit full conformal invariance in the absence of the usual Einstein-Hilbert term in the action, introducing additionally either the Weyl derivative or properly rescaled fields $[35,50]$. Such a construction corresponds to a higher-order version of the usual conformal coupling, first studied by Bocharova, Bronnikov and Melnikov in [51] and independently investigated by Bekenstein in [52], namely the BBMB action (see also [53-56]). In this theory, one considers an additional Galileon field, allowing for a nonminimal derivative coupling [57] with the Einstein tensor and the energy momentum tensor of the first, conformally coupled scalar field [58], without a potential i.e. maintaining the shift symmetry of the additional scalar. The black-hole application of this theory leads to the interesting result of the presence of a primary scalar hair [50] (see [59] regarding possible black-hole instability issues in shift-symmetric theories).

In this work we are interested in investigating the cosmological implications of the above bi-scalar theory with (partial) conformal invariance. In particular, we desire to extract analytical solutions and study the late-time evolution of a universe governed by such a gravitational modification. The plan of the manuscript is the following: In Section 2 we present the construction of the theory, while in Section 3 we apply it in a cosmological framework, giving the relevant equations. Then, in Section 4 we extract various analytical solutions, as well as we perform a numerical elaboration of the full cosmological system. Finally, in 5 we summarize the obtained results. 


\section{Action and field equations}

In this section we demonstrate how to gradually build the action of the particular bi-scalar theory, which parts of it are conformally invariant, and then we extract the general field equations. For more information on the procedure, one can look at [50]. We start from the usual conformally-coupled action, often called Bocharova-Bronnikov-Melnikov-Bekenstein (BBMB) action [51, 52] which we denote it as $S_{0}$, since it is going to represent the "seed" action, namely

$$
S_{0}=\int d x^{4} \sqrt{-g}\left\{\frac{1}{16 \pi G} R+\eta\left[-\frac{1}{2}(\partial \phi)^{2}-\frac{1}{12} \phi^{2} R\right]\right\},
$$

where $G$ is the Newton's constant. In the above action apart from the Einstein Hilbert term, an additional scalar field $\phi$, conformally coupled to the Ricci scalar $R$, has also been considered, and we have introduced the dimension-less coupling parameter $\eta$. As one can easily see the term in the brackets is invariant under the transformation [58]

$$
\begin{aligned}
& g_{\mu \nu} \rightarrow \Omega(x)^{2} g_{\mu \nu}, \\
& \phi \rightarrow \Omega(x)^{-1} \phi,
\end{aligned}
$$

however $S_{0}$ is not fully conformally invariant due to the presence of the usual $R$-term. Varying the first part of the action with respect to the metric provides the Einstein tensor, while varying the second term provides the energy-momentum tensor of a conformallycoupled scalar field, which in four dimensions reads

$$
T_{\mu \nu}^{(\phi)}=\frac{1}{2} \nabla_{\mu} \phi \nabla_{\nu} \phi-\frac{1}{4} g_{\mu \nu} \nabla_{\alpha} \phi \nabla^{\alpha} \phi+\frac{1}{12}\left(g_{\mu \nu} \square-\nabla_{\mu} \nabla_{\nu}+G_{\mu \nu}\right) \phi^{2} .
$$

Let us now try to include higher-order terms in the action $S_{0}$, introducing additionally a new degree of freedom. For this shake, a second scalar $\Psi$ is added, allowing also for a non-minimally derivative coupling with the Einstein tensor, and moreover an additional coupling of this second scalar with the energy-momentum tensor of the conformally-coupled scalar $\phi$. Hence, we consider an additional action $S_{1}$ as

$$
S_{1}=\int d x^{4} \sqrt{-g}\left[\beta G_{\mu \nu} \nabla^{\mu} \Psi \nabla^{\nu} \Psi-\gamma T_{\mu \nu}^{(\phi)} \nabla^{\mu} \Psi \nabla^{\nu} \Psi\right],
$$

where $\beta$ and $\gamma$ are dimension-full coupling constants. Thus, in summary, we will consider the total action

$$
S=S_{0}+S_{1}
$$

The reason for considering such an action is the following: The $\Psi$-field equation (obtained from (2.5)), due to shift symmetry, can be nicely written as a current conservation equation, namely

$$
\mathcal{E}_{\Psi}=\nabla_{\mu} J^{\mu}=0
$$


with

$$
J^{\mu}=-2\left(\beta G^{\mu \nu}-\gamma T^{\mu \nu}\right) \nabla_{\nu} \Psi
$$

Therefore, it becomes obvious that the current vector $J^{\mu}$ "encodes" the metric field equations of the action (2.1) (this would become impossible if the sift symmetry was broken, for instance by including a general potential). That is why we can refer to the action $S_{0}$ as being the "seed" action, or the precursor, of the higher-order action $S_{1}$.

With this procedure, at the level of the action, an already existing theory can be extended by picking up its field equations and coupling it accordingly with an additional field. The resulting theory is now a bi-scalar tensor theory [28, 31], however the important advantage is that it is also partially conformally invariant $[35,58]$, and, similarly to the seed theory, it can also become fully conformally invariant if one neglects the first, EinsteinHilbert term, and introduces either the Weyl derivative or properly rescaled fields [35, 50]. Furthermore, due to this construction method, the field equations are ensured that they will not contain more than two time derivatives, which is an additional significant advantage. One could follow the above procedure on and on, inserting additional scalars, resulting to partially conformally invariant multi-scalar theories. However in this work we desire to remain in the bi-scalar case for simplicity.

Variation of the action (2.6) with respect to the metric leads to

$$
\begin{aligned}
& \mathcal{H}_{\mu \nu}=\frac{1}{16 \pi G} G_{\mu \nu}-\eta\left[\frac{1}{2} \nabla_{\mu} \phi \nabla_{\nu} \phi-\frac{1}{4} g_{\mu \nu} \nabla_{\alpha} \phi \nabla^{\alpha} \phi+\frac{1}{12}\left(g_{\mu \nu} \square-\nabla_{\mu} \nabla_{\nu}+G_{\mu \nu}\right) \phi^{2}\right] \\
&+\beta\left\{-\frac{1}{2} g_{\mu \nu} G^{\alpha \beta} \nabla_{\alpha} \Psi \nabla_{\beta} \Psi+2 G_{(\mu}{ }^{\lambda} \nabla_{\nu)} \Psi \nabla_{\lambda} \Psi+\frac{1}{2} R \nabla_{\mu} \Psi \nabla_{\nu} \Psi-\frac{1}{2} R_{\mu \nu} \nabla^{\alpha} \Psi \nabla_{\alpha} \Psi\right. \\
& \quad+\frac{1}{2} g_{\mu \nu}\left[(\square \Psi)^{2}-\nabla_{\alpha} \nabla_{\beta} \Psi \nabla^{\alpha} \nabla^{\beta} \Psi-R_{\alpha \beta} \nabla^{\alpha} \Psi \nabla^{\beta} \Psi\right] \\
&\left.\quad+\nabla_{\mu} \nabla^{\alpha} \Psi \nabla_{\nu} \nabla_{\alpha} \Psi-\square \Psi \nabla_{\mu} \nabla_{\nu} \Psi+R_{\mu}^{\alpha}{ }_{\nu}^{\beta} \nabla_{\alpha} \Psi \nabla_{\beta} \Psi\right\} \\
&+\gamma\left\{\frac{1}{4} g_{\mu \nu} \nabla_{\alpha} \phi \nabla_{\beta} \phi \nabla^{\alpha} \Psi \nabla^{\beta} \Psi-\nabla_{(\mu} \phi \nabla_{\nu)} \Psi \nabla_{\alpha} \phi \nabla^{\alpha} \Psi-\frac{1}{8} g_{\mu \nu} \nabla_{\alpha} \phi \nabla^{\alpha} \phi \nabla_{\beta} \Psi \nabla^{\beta} \Psi\right. \\
& \quad+\frac{1}{4} \nabla_{\mu} \phi \nabla_{\nu} \phi \nabla_{\beta} \Psi \nabla^{\beta} \Psi+\frac{1}{4} \nabla_{\alpha} \phi \nabla^{\alpha} \phi \nabla_{\mu} \Psi \nabla_{\nu} \Psi+\frac{1}{24} g_{\mu \nu} \square\left(\phi^{2}\right) \nabla_{\alpha} \Psi \nabla^{\alpha} \Psi \\
& \quad-\frac{1}{12} \nabla_{\mu} \nabla_{\nu} \phi^{2} \nabla_{\alpha} \Psi \nabla^{\alpha} \Psi+\frac{1}{12} \nabla_{(\mu}\left[\nabla_{\nu)}\left(\phi^{2}\right) \nabla_{\alpha} \Psi \nabla^{\alpha} \Psi\right]-\frac{1}{24} g_{\mu \nu} \nabla^{\beta}\left(\nabla_{\beta} \phi^{2} \nabla_{\alpha} \Psi \nabla^{\alpha} \Psi\right) \\
& \quad-\frac{1}{12} \square\left(\phi^{2}\right) \nabla_{\mu} \Psi \nabla_{\nu} \Psi-\frac{1}{24} g_{\mu \nu} \nabla_{\alpha} \nabla_{\beta} \phi^{2} \nabla^{\alpha} \Psi \nabla^{\beta} \Psi+\frac{1}{6} \nabla_{\alpha} \nabla_{(\mu} \phi^{2} \nabla_{\nu)} \Psi \nabla^{\alpha} \Psi \\
& \quad-\frac{1}{12} \nabla_{\alpha}\left[\nabla_{(\mu} \phi^{2} \nabla_{\nu)} \Psi \nabla^{\alpha} \Psi\right]+\frac{1}{24} \nabla_{\alpha}\left(\nabla^{\alpha} \phi^{2} \nabla_{\mu} \Psi \nabla_{\nu} \Psi\right)+\frac{1}{24} \phi^{2} g_{\mu \nu} G^{\alpha \beta} \nabla_{\alpha} \Psi \nabla_{\beta} \Psi \\
&-\frac{1}{6} \phi^{2} G_{(\mu}{ }^{\lambda} \nabla_{\nu)} \Psi \nabla_{\lambda} \Psi-\frac{1}{24} \phi^{2} R \nabla_{\mu} \Psi \nabla_{\nu} \Psi+\frac{1}{24} \phi^{2} R_{\mu \nu} \nabla^{\alpha} \Psi \nabla_{\alpha} \Psi \\
&+\frac{1}{12} \square \Psi \nabla_{(\mu}\left(\phi^{2}\right) \nabla_{\nu)} \Psi-\frac{1}{12} \nabla^{\alpha} \Psi \nabla_{(\mu}\left(\phi^{2}\right) \nabla_{\nu)} \nabla_{\alpha} \Psi-\frac{1}{12} \nabla_{\alpha}\left(\phi^{2}\right) \nabla_{(\mu} \nabla^{\alpha} \Psi \nabla_{\nu)} \Psi \\
&+\frac{1}{12} \nabla_{\alpha}\left(\phi^{2}\right) \nabla^{\alpha} \Psi \nabla_{\mu} \nabla_{\nu} \Psi-\frac{1}{24} \square\left(\phi^{2}\right) \nabla_{\mu} \Psi \nabla_{\nu} \Psi-\frac{1}{24} \nabla_{\mu} \nabla_{\nu}\left(\phi^{2}\right) \nabla^{\alpha} \Psi \nabla_{\alpha} \Psi \\
&-\frac{1}{24} g_{\mu \nu} \nabla_{\alpha} \nabla_{\beta}\left(\phi^{2}\right) \nabla^{\alpha} \Psi \nabla^{\beta} \Psi-\frac{1}{12} g_{\mu \nu} \nabla_{\alpha}\left(\phi^{2}\right) \nabla^{\alpha} \Psi \square \Psi+\frac{1}{12} g_{\mu \nu} \nabla_{\alpha}\left(\phi^{2}\right) \nabla_{\beta} \Psi \nabla^{\alpha} \nabla^{\beta} \Psi
\end{aligned}
$$




$$
\begin{aligned}
&+\frac{1}{12} \nabla_{\alpha} \nabla_{(\mu}\left(\phi^{2}\right) \nabla^{\alpha} \Psi \nabla_{\nu)} \Psi+\frac{1}{24} g_{\mu \nu} \square\left(\phi^{2}\right) \nabla^{\alpha} \Psi \nabla_{\alpha} \Psi \\
&-\frac{1}{12} \phi^{2}\left\{\frac{1}{2} g_{\mu \nu}\left[(\square \Psi)^{2}-\nabla_{\alpha} \nabla_{\beta} \Psi \nabla^{\alpha} \nabla^{\beta} \Psi-R_{\alpha \beta} \nabla^{\alpha} \Psi \nabla^{\beta} \Psi\right]+\nabla_{\mu} \nabla^{\alpha} \Psi \nabla_{\nu} \nabla_{\alpha} \Psi\right. \\
&\left.\left.-\square \Psi \nabla_{\mu} \nabla_{\nu} \Psi+R_{\mu}{ }^{\alpha}{ }_{\nu}{ }^{\beta} \nabla_{\alpha} \Psi \nabla_{\beta} \Psi\right\}\right\}=0
\end{aligned}
$$

where the parentheses in space-time indices denote symmetrization.

Furthermore, variation of $(2.6)$ with respect to $\phi$ leads to

$$
\begin{aligned}
& \mathcal{E}_{\phi}= \eta\left(\square \phi-\frac{1}{6} R \phi\right) \\
&+\gamma\left[\frac{5}{6} \nabla_{\nu}\left(\nabla^{\nu} \Psi \nabla_{\mu} \phi \nabla^{\mu} \Psi\right)-\frac{1}{3} \nabla^{\alpha}\left(\nabla_{\alpha} \phi \nabla_{\mu} \Psi \nabla^{\mu} \Psi\right)-\frac{1}{6} \square \phi \nabla_{\mu} \Psi \nabla^{\mu} \Psi\right. \\
&+\frac{1}{6} \nabla_{\mu} \nabla_{\nu} \phi \nabla^{\mu} \Psi \nabla^{\nu} \Psi-\frac{1}{6} \phi G_{\mu \nu} \nabla^{\mu} \Psi \nabla^{\nu} \Psi+\frac{1}{6} \square \Psi \nabla_{\nu} \phi \nabla^{\nu} \Psi+\frac{1}{6} \phi(\square \Psi)^{2} \\
&\left.\quad-\frac{1}{6} \nabla_{\nu} \phi \nabla^{\mu} \Psi \nabla_{\mu} \nabla^{\nu} \Psi-\frac{1}{6} \phi \nabla_{\mu} \nabla_{\nu} \Psi \nabla^{\mu} \nabla^{\nu} \Psi-\frac{1}{6} \phi R_{\mu \nu} \nabla^{\mu} \Psi \nabla^{\nu} \Psi\right]=0 .
\end{aligned}
$$

Finally, as we have already mentioned, variation of the action (2.6) with respect to $\Psi$ leads to equation (2.7), which is its equation of motion.

\section{Cosmology}

In the previous section we demonstrated how to built a bi-scalar theory that maintains the conformal invariance of the "seed" single-scalar theory (up to the Einstein-Hilbert term). In this section we are interested in applying this theory at a cosmological framework. Hence, we consider a flat Friedmann-Robertson-Walker (FRW) spacetime metric of the form

$$
d s^{2}=-d t^{2}+a(t)^{2} \delta_{i j} d x^{i} d x^{j},
$$

with $a(t)$ the scale factor. Additionally, we consider the matter sector, described by a perfect-fluid action $S_{m}$, and thus the total action will be

$$
S=S_{0}+S_{1}+S_{m}
$$

In this case, variation with respect to the metric leads to the metric-field equations

$$
\mathcal{H}_{\mu \nu}=\frac{1}{2} T_{\mu \nu}^{(m)},
$$

with $\mathcal{H}_{\mu \nu}$ given by (2.9) and where $T_{\mu \nu}^{(m)}=\frac{-2}{\sqrt{-g}} \frac{\delta S_{m}}{\delta g^{\mu \nu}}$ is the energy-momentum tensor of the matter perfect fluid. In particular, in the case of FRW geometry, they write explicitly as

$$
\frac{3}{8 \pi G} H^{2}-\rho_{m}-\frac{\eta}{2}(H \phi+\dot{\phi})^{2}-9 \beta H^{2} \dot{\Psi}^{2}+\frac{3 \gamma}{4}(H \phi+\dot{\phi})^{2} \dot{\Psi}^{2}=0
$$




$$
\begin{aligned}
\frac{1}{8 \pi G}( & \left.3 H^{2}+2 \dot{H}\right)+p_{m}+\frac{\eta}{6}\left[\dot{\phi}^{2}-3 H^{2} \phi^{2}-4 H \phi \dot{\phi}-2 \phi(\phi \dot{H}+\ddot{\phi})\right] \\
& -\beta \dot{\Psi}\left[\left(3 H^{2}+2 \dot{H}\right) \dot{\Psi}+4 H \ddot{\Psi}\right] \\
& +\frac{\gamma}{12} \dot{\Psi}\left\{\dot{\Psi}\left[3 H^{2} \phi^{2}+4 H \phi \dot{\phi}-\dot{\phi}^{2}+2 \phi(\phi \dot{H}+\ddot{\phi})\right]+4 \phi(H \phi+\dot{\phi}) \ddot{\Psi}\right\}=0,
\end{aligned}
$$

which are just the Friedmann equations of the scenario at hand. In the above expressions dots denote differentiation with respect to $t, H=\dot{a} / a$ is the Hubble parameter, and $\rho_{m}$ and $p_{m}$ are respectively the matter energy density and pressure. Similarly, the two scalar field equations (2.10) and (2.7) in the case of FRW geometry become:

$$
\mathcal{E}_{\phi}=-\frac{1}{2}\left(2 \eta-\gamma \dot{\Psi}^{2}\right)\left[\phi\left(2 H^{2}+\dot{H}\right)+3 H \dot{\phi}+\ddot{\phi}\right]+\gamma(H \phi+\dot{\phi}) \dot{\Psi} \ddot{\Psi}=0,
$$

and

$$
\begin{aligned}
\mathcal{E}_{\Psi}= & 6 \beta H\left[\left(3 H^{2}+2 \dot{H}\right) \dot{\Psi}+H \ddot{\Psi}\right] \\
& -\frac{1}{2} \gamma(H \phi+\dot{\phi})\left\{\dot{\Psi}\left[3 H^{2} \phi+5 H \dot{\phi}+2(\phi \dot{H}+\ddot{\phi})\right]+(H \phi+\dot{\phi}) \ddot{\Psi}\right\}=0 .
\end{aligned}
$$

Finally, one can straightforwardly verify that the above equations satisfy the equations arising from the fact that the total action is diffeomorphism invariant [31]:

$$
\nabla_{\mu} \mathcal{H}^{\mu \nu}+\frac{1}{2} \mathcal{E}_{\phi} \nabla^{\nu} \phi+\frac{1}{2} \mathcal{E}_{\Psi} \nabla^{\nu} \Psi=\frac{1}{2} \nabla_{\mu} T^{\mu \nu}=0
$$

Lastly, we stress that the above equations do not contain higher than two time-derivatives, as expected due to the construction method we followed in order to build this conformal bi-scalar scenario.

Concerning the late-time application of the above equations, we can immediately see that we can re-write the two Friedmann equations $(3.4),(3.5)$ in the usual form, namely

$$
\begin{aligned}
& H^{2}=\frac{8 \pi G}{3}\left(\rho_{D E}+\rho_{m}\right) \\
& 2 \dot{H}+3 H^{2}=-8 \pi G\left(p_{D E}+p_{m}\right),
\end{aligned}
$$

if we define an effective dark energy sector with energy density and pressure respectively as:

$$
\begin{aligned}
\rho_{D E} \equiv & \frac{\eta}{2}(H \phi+\dot{\phi})^{2}+9 \beta H^{2} \dot{\Psi}^{2}-\frac{3 \gamma}{4}(H \phi+\dot{\phi})^{2} \dot{\Psi}^{2}, \\
p_{D E} \equiv & \frac{\eta}{6}\left[\dot{\phi}^{2}-3 H^{2} \phi^{2}-4 H \phi \dot{\phi}-2 \phi(\phi \dot{H}+\ddot{\phi})\right] \\
& -\beta \dot{\Psi}\left[\left(3 H^{2}+2 \dot{H}\right) \dot{\Psi}+4 H \ddot{\Psi}\right] \\
& +\frac{\gamma}{12} \dot{\Psi}\left\{\dot{\Psi}\left[3 H^{2} \phi^{2}+4 H \phi \dot{\phi}-\dot{\phi}^{2}+2 \phi(\phi \dot{H}+\ddot{\phi})\right]+4 \phi(H \phi+\dot{\phi}) \ddot{\Psi}\right\} .
\end{aligned}
$$

Thus, in the scenario at hand we acquire an effective dark-energy sector that consists of both scalar fields. Additionally, using their equations of motion (3.6) and (3.7), we can verify that

$$
\dot{\rho}_{D E}+3 H\left(\rho_{D E}+p_{D E}\right)=0
$$


and we can define the equation-of-state parameter for the effective dark-energy sector as

$$
w_{D E} \equiv \frac{p_{D E}}{\rho_{D E}} .
$$

Lastly, we mention that as usual the matter energy density and pressure satisfy the equation

$$
\dot{\rho}_{m}+3 H\left(\rho_{m}+p_{m}\right)=0 .
$$

Before proceeding, it is worthy to mention that the equations of motion for the scalar fields $\phi$ and $\Psi$ admit a first integral. In particular, (3.6) can be written as

$$
2 a^{2} \mathcal{E}_{\phi}=-\partial_{t}\left[\left(2 \eta-\gamma \dot{\Psi}^{2}\right) a(\phi \dot{a}+a \dot{\phi})\right],
$$

while (3.7) can be written as

$$
a^{3} \mathcal{E}_{\Psi}=\partial_{t}\left[a^{3} J^{0}\right]=0
$$

with

$$
J_{0}=\left[\left(-12 \beta+\gamma \phi^{2}\right) \dot{a}^{2}+2 \gamma a \phi \dot{a} \dot{\phi}+\gamma a^{2} \dot{\phi}^{2}\right] \dot{\Psi},
$$

and hence we respectively obtain:

$$
\begin{aligned}
& \left(2 \eta-\gamma \dot{\Psi}^{2}\right) a(\phi \dot{a}+a \dot{\phi})=c_{0} \\
& -6 \beta a \dot{a}^{2} \dot{\Psi}+\frac{1}{2} \gamma a(\phi \dot{a}+a \dot{\phi})^{2} \dot{\Psi}=c_{1},
\end{aligned}
$$

where $c_{0}, c_{1}$ are integration constants. These expressions are going to be crucial in the following section, since they allow us to extract analytical solutions.

\section{Solutions}

In the previous section we derived the cosmological equations in a bi-scalar model which exhibits partial conformal invariance. In this section we are first interested in extracting analytical solutions in the case where the matter sector is absent, and moreover to investigate the scenario numerically in the case where matter is present. As we mentioned above, the equations of motion for the scalar fields admit the first integrals (3.19),(3.20), which proves to be crucial in the solution extraction.

\subsection{Case 1: $c_{0}=0$}

Let us first neglect the matter sector and investigate the case where the first integral of the $\phi$-field equation, i.e. (3.19), is equal to zero, namely we consider $c_{0}=0$. Hence, we acquire two subcases, corresponding to which of the two parentheses terms becomes zero.

- In the first subcase, namely when the first parenthesis in (3.19) is zero, we have that

$$
2 \eta-\gamma \dot{\Psi}^{2}=0,
$$


which admits the solution

$$
\Psi(t)= \pm \sqrt{\frac{2 \eta}{\gamma}} t+\Psi_{0}
$$

with $\Psi_{0}$ an integration constant. Both sign-branches lead to the same observable results, since both the Friedmann equations (3.4),(3.5), as well as the scalar-field equations (3.6), (3.7) depend only on $\dot{\Psi}^{2}$ and/or $\dot{\Psi} \ddot{\Psi}$. Substituting the above solution (4.2) for $\Psi(t)$ into the second Friedmann equation (3.5) we obtain

$$
\left(\dot{a}^{2}+2 a \ddot{a}\right)(\gamma-16 \pi G \beta \eta)=0 .
$$

If the first bracket of (4.3) is zero, namely if $\dot{a}^{2}+2 a \ddot{a}=0$, then we immediately extract the solution

$$
a(t)=a_{0}\left(t-t_{0}\right)^{2 / 3},
$$

where $a_{0}, t_{0}$ are integrations constant. Thus, substituting the above $\Psi(t)$ and $a(t)$ into the first Friedmann equation (3.4) we finally acquire the solution for $\phi$ as

$$
\phi(t)=\frac{\phi_{0}}{\left(t-t_{0}\right)^{2}} \pm \frac{\sqrt{6} \sqrt{3 \beta \eta-\gamma / 16 \pi G}}{\sqrt{\gamma \eta}},
$$

with $\phi_{0}$ an integration constant. Note that the constant $t_{0}$ can be set to zero without loss of generality.

Interestingly enough, we observe that in the above solution the universe behaves as a matter-era although we have not explicitly considered the matter sector. This can be immediately explained, since in this case the "effective" dark energy sector constituted from the two scalar fields obtains an equation-of-state parameter equal to zero, as can be seen from (3.14). Hence, in the scenario at hand we have obtained a form of "mimetic" dark matter [60,61], constituted from the two scalar fields. This important feature deserves further investigation, in particular examining the behavior of this solution under perturbations and confronting it with observational data from large-scale structure [62]. Such a project is left for a future investigation.

If now the second bracket of (4.3) is zero, namely $\gamma-16 \pi G \beta \eta=0$, then the first Friedmann equation (3.4) gives

$$
\phi(t)=\frac{\phi_{0}}{a(t)} \pm 2 \sqrt{\frac{3 \beta}{\gamma}} .
$$

Substituting this solution into the field equation (3.7) for $\Psi(t)$ we see that it is immediately satisfied, which was expected since in this case $J^{0}$ in (3.17) is identically zero. Hence, this solution branch is compatible with every cosmological evolution $a(t)$. This is a important, since one can obtain arbitrary scale-factor behavior with only tuning the parameters as required for this branch, namely $c_{0}=0$ and $\gamma-$ $16 \pi G \beta \eta=0$. This feature is a significant advantage of the scenario at hand, since 
apart from late-time acceleration one can describe the complete thermal history of the universe, namely the sequence from inflation, to radiation, to matter and to darkenergy epochs, as well as alternatively evolutions such as the bouncing or cyclic ones [63].

- In the second subcase, namely when the second parenthesis in (3.19) is zero, we have that

$$
\phi \dot{a}+a \dot{\phi}=0,
$$

which leads to the solution

$$
\phi(t)=\frac{\phi_{0}}{a(t)}
$$

Substituting into the first Friedmann equation (3.4) we obtain

$$
\Psi(t)= \pm \sqrt{\frac{1}{24 \pi G \beta}} t+\Psi_{0},
$$

with $\Psi_{0}$ an integration constant. Moreover, inserting these into the second Friedmann equation (3.5) we acquire $\dot{a}^{2}+2 a \ddot{a}=0$, which admits the solution

$$
a(t)=a_{0}\left(t-t_{0}\right)^{2 / 3} .
$$

Finally, substituting this into relation (4.8) we obtain:

$$
\phi(t)=\frac{\phi_{0}}{a_{0}\left(t-t_{0}\right)^{2 / 3}} .
$$

Similarly to the previous subcase, we can see that we obtain a matter era despite the fact that we have not considered an explicit matter sector, namely we obtain a mimetic dark matter arising from the combination of the two coupled scalar fields. This is a great advantage of the scenario at hand.

\subsection{Case 2: $16 \pi G \eta \beta-\gamma=0$}

Let us now investigate another class of solutions, characterized by the parameter relation $16 \pi G \eta \beta-\gamma=0$ (note that in contrast with the second branch of the first subcase of the previous subsection we keep a general $c_{0}$ ), still without considering an explicit matter sector. In this case the first Friedmann equation (3.4) acquires the factorized form

$$
\left(3 \beta \dot{\Psi}^{2}-\frac{1}{8 \pi G}\right)\left[12 \beta \dot{a}^{2}-\gamma(\phi \dot{a}+a \dot{\phi})^{2}\right]=0
$$

- If the first bracket of (4.12) is zero then $\Psi(t)$ obtains the linear relation (4.9), namely

$$
\Psi(t)= \pm \sqrt{\frac{1}{24 \pi G \beta}} t+\Psi_{0},
$$


however in this case the first integral (3.19) leads to

$$
\phi(t)=\frac{1}{a(t)}\left[\phi_{0}+\frac{12 \pi G c_{0} \beta}{\gamma} \int \frac{d t^{\prime}}{a\left(t^{\prime}\right)}\right] .
$$

Hence, substituting these into the second Friedmann equation (3.5) we can easily obtain

$$
H^{2}=\frac{12 \pi^{2} G^{2} c_{0}^{2} \beta}{\gamma a^{4}}+\frac{C_{1}}{a^{3}}
$$

where $C_{1}$ is a new integration constant. Interestingly enough, we observe that in this subclass of solutions we obtain in the Friedmann equation both an effective dark matter sector, as well as an effective dark radiation sector $[64,65]$, despite the fact that we have not considered explicitly such sectors. This is a great advantage of the scenario, since the two coupled scalar fields can give rise to these effective sectors in a mimetic way. This feature could have very interesting physical implications.

The solution of (4.15) is straightforward, it is a fractional function of $t$, and since it exists in the literature [66] we do not write it explicitly here. We just mention that in the case where $c_{0}=0$, i.e. when the effective dark radiation disappears, we obtain the usual matter era evolution, namely $a(t)=(3 / 2)^{2 / 3} C_{1}^{1 / 3}\left(t-t_{0}\right)^{2 / 3}$, while in the case where $C_{1}=0$, i.e. when the effective dark matter disappears, we obtain the usual radiation era evolution, namely $a(t)=\sqrt{2}\left(\frac{12 \pi^{2} G^{2} c_{0}^{2} \beta}{\gamma}\right)^{1 / 4}\left(t-t_{0}\right)^{1 / 2}$. However, in general, using (4.15) one can reconstruct the observed thermal history of the universe, with an initial radiation era (for small scale factors) followed by the matter epoch (for larger scale factors).

- If now the second bracket of (4.12) is zero then $\phi(t)$ obtains the solution (4.6), namely

$$
\phi(t)=\frac{\phi_{0}}{a(t)} \pm 2 \sqrt{\frac{3 \beta}{\gamma}},
$$

however in this case the first integral (3.19) leads to

$$
\Psi(t)=\int \sqrt{\frac{1}{8 \pi G \beta}-\frac{c_{0}}{2 \sqrt{3 \beta \gamma} a \dot{a}}} d t+\Psi_{0} .
$$

Note that the above two solutions of $\phi(t)$ and $\Psi(t)$ with respect to $a(t)$ satisfy all the field equations for arbitrary scale factor. Hence, this solution subclass can reproduce any cosmological evolution, which is a significant advantage of the scenario at hand, revealing its capabilities.

\subsection{General case}

Let us now investigate the general solution subclass without the presence of an explicit matter sector. The cosmological system consists of the two Friedmann equations (3.4),(3.5) 
and the two scalar field equations expressed as integrals, namely (3.19),(3.20), with only three of them being independent. Considering for $\phi(t)$ the ansatz

$$
\phi(t)=\frac{k(t)}{a(t)}
$$

then $(3.4),(3.19),(3.20)$ respectively become

$$
\begin{aligned}
\dot{a}^{2}\left(36 \beta \dot{\Psi}^{2}-\frac{3}{2 \pi G}\right)+\dot{k}^{2}\left(2 \eta-3 \gamma \dot{\Psi}^{2}\right) & =0 \\
a \dot{k}\left(2 \eta-\gamma \dot{\Psi}^{2}\right)-c_{0} & =0 \\
\frac{1}{2} a\left(\gamma \dot{k}^{2}-12 \beta \dot{a}^{2}\right) \dot{\Psi}-c_{1} & =0 .
\end{aligned}
$$

- In the trivial case where $36 \beta \dot{\Psi}^{2}-\frac{3}{2 \pi G}=0$, which implies that $\Psi(t)=\sqrt{\frac{1}{24 \pi G \beta}} t+\Psi_{0}$, Eq. (4.19) leads either to $16 \pi G \eta \beta-\gamma=0$, case which was examined in the previous subsection, or to $\dot{k}=0$, which leads to

$$
\phi(t)=\frac{\phi_{0}}{a(t)} .
$$

Substituting in (4.20),(4.21) we see that the full system is satisfied if

$$
a(t)=a_{0}\left(t-t_{0}\right)^{2 / 3},
$$

with the additional parameter constraint $9 \sqrt{\pi G} c 1+2 \sqrt{6} a_{0}^{3} \sqrt{\beta}=0$. Once again we observe that we obtain a matter era, although we have not considered an explicit matter sector, due to the fact that the two scalars produce an effective matter sector of mimetic nature.

- In the general case where $36 \beta \dot{\Psi}^{2}-\frac{3}{2 \pi G} \neq 0$, Eq. (4.19) leads to

$$
\dot{a}= \pm \dot{k} \sqrt{\frac{\left(3 \gamma \dot{\Psi}^{2}-2 \eta\right)}{\left(36 \beta \dot{\Psi}^{2}-\frac{3}{2 \pi G}\right)}},
$$

where we have assumed that $\dot{\Psi}^{2}$ lies in the appropriate ranges for the above relation to hold, namely

$$
\begin{array}{lll}
\frac{3}{72 \pi G \beta}<\dot{\Psi}^{2} \leq \frac{2 \eta}{3 \gamma} & \text { if } & \frac{2 \eta}{\gamma}<\frac{1}{8 \pi G \beta} \\
\frac{2 \eta}{3 \gamma} \leq \dot{\Psi}^{2}<\frac{3}{72 \pi G \beta} & \text { if } & \frac{2 \eta}{\gamma}>\frac{1}{8 \pi G \beta} .
\end{array}
$$

Substituting (4.24) into (4.21) we obtain

$$
(\gamma-16 \pi G \beta \eta) a \dot{k}^{2} \dot{\Psi}=2 c_{1}\left(1-24 \pi G \beta \dot{\Psi}^{2}\right) .
$$

Hence, disregarding the case where $16 \pi G \eta \beta-\gamma=0$ analyzed in the previous subsection, or the trivial cases $\dot{\Psi}=0$ which leads to an effective radiation era of the form 
$a(t)=a_{0}\left(t-t_{0}\right)^{1 / 2}$, and $\dot{k}=0$ which leads to an effective matter era of the form $a(t)=a_{0}\left(t-t_{0}\right)^{2 / 3}$, Eq. (4.27) leads to

$$
a(t)=\frac{2 c_{1}\left(1-24 \pi G \beta \dot{\Psi}^{2}\right)}{(\gamma-16 \pi G \beta \eta) \dot{k}^{2} \dot{\Psi}} .
$$

Inserting this expression into (4.20) we acquire

$$
\dot{k}=\frac{2 c_{1}\left(1-24 \pi G \beta \dot{\Psi}^{2}\right)\left(2 \eta-\gamma \dot{\Psi}^{2}\right)}{c_{0}(\gamma-16 \pi G \beta \eta) \dot{\Psi}},
$$

and finally combing (4.24), with (4.28) and (4.29) we arrive on a master equation

$$
\begin{aligned}
& \frac{c_{0}^{4}\left(\frac{\gamma}{16 \pi G}-\beta \eta\right)^{2}\left[\frac{\eta}{4 \pi G}+6\left(\frac{\gamma}{16 \pi G}+\beta \eta\right) \dot{\Psi}^{2}-15 \beta \gamma \dot{\Psi}^{4}\right]^{2} \ddot{\Psi}^{2}}{c_{1}^{2}\left(\frac{1}{8 \pi G}-3 \beta \dot{\Psi}^{2}\right)^{4}\left(\gamma \dot{\Psi}^{2}-2 \eta\right)^{6}} \\
& +\frac{c_{1}^{2}\left(\frac{1}{8 \pi G}-3 \beta \dot{\Psi}^{2}\right)\left(\gamma \dot{\Psi}^{2}-2 \eta\right)\left(3 \gamma \dot{\Psi}^{2}-2 \eta\right)}{12 c_{0}^{2}\left(\frac{\gamma}{16 \pi G}-\beta \eta\right)^{2} \dot{\Psi}^{2}}=0 .
\end{aligned}
$$

The solution for $\Psi(t)$ of this equation allows us to go back and extract the solutions for the remaining involved quantities, namely $a(t)$ and $\phi(t)$. Unfortunately, the above equations cannot be solved analytically in general. Hence, one should either investigate the subcases that we have already studied in the previous subsections, or solve the full equation approximately expanding it and keeping the lower orders, or solve it numerically. However, since in the following subsection we will elaborate numerically the full cosmological equations, namely including the explicit matter sector, we are not interested in solving numerically (4.30), which corresponds to the subclass without matter.

\subsection{Numerical elaboration}

In the previous subsections we presented analytical solutions of the cosmological system at hand, in the case where an explicit matter sector is absent. In this subsection we will investigate the full cosmological equations, including the matter sector, namely equations (3.4),(3.5),(3.6), (3.7), focusing at late times. Since these equations cannot be solved analytically, we will perform a numerical elaboration.

As usual, we assume that the explicit matter sector corresponds to a dust fluid, namely we consider $p_{m} \approx 0$. Furthermore, in order for our cosmological evolution to be consistent with observations, we impose the present values of the density parameters to be $\Omega_{m 0}=$ $\frac{8 \pi G \rho_{m 0}}{3 H^{2}} \approx 0.3$ and $\Omega_{D E 0}=\frac{8 \pi G \rho_{D E 0}}{3 H^{2}} \approx 0.7$ [38]. Finally, we use the redshift $z=-1+a_{0} / a$ as the independent variable, setting the current scale factor $a_{0}$ to 1 .

In Fig. 1 we show the cosmological evolution for the parameters choice $\eta=1, \beta=-11$, $\gamma=-1$ in units where $8 \pi G=1$, focusing on various observables. Specifically, in the upper graph we present the evolution of the matter and dark energy density parameters, defined as $\Omega_{i}=8 \pi G \rho_{i} /\left(3 H^{2}\right)$, and as we can see it is in agreement with the observed one [38]. 
In the middle graph of Fig. 1 we depict the behavior of the dark-energy equation-of-state parameter $w_{D E}$, which presents a dynamical behavior, acquiring at present a value very close to the cosmological-constant one, as expected from observations. Finally, in the lower graph of Fig. 1 we present the evolution of the deceleration parameter $q=-1-\dot{H} / H^{2}$. As we observe, the universe passed from deceleration $(q>0)$ to acceleration $(q<0)$ in the recent cosmological past, as it is required from observations. In summary, the examined

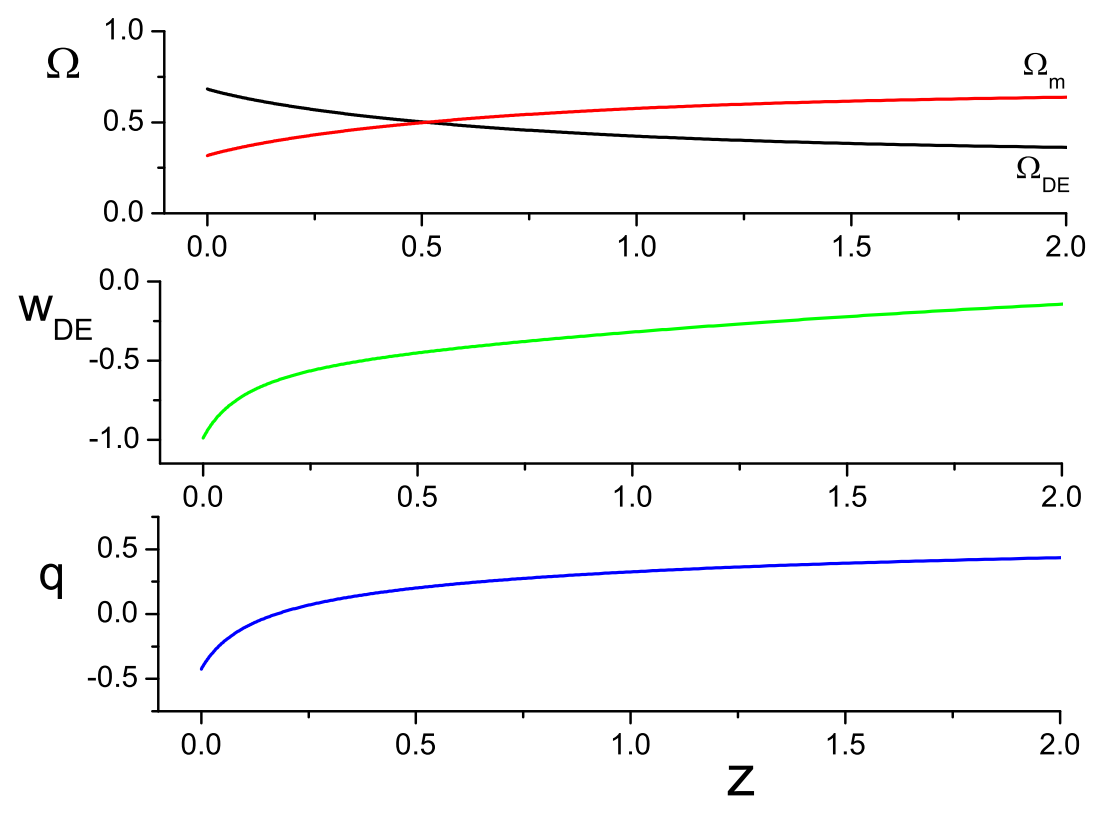

Figure 1. The late-time cosmological evolution, for the parameters choice $\eta=1, \beta=-11, \gamma=-1$ in units where $8 \pi G=1$, having imposed $\Omega_{m 0} \approx 0.3, \Omega_{D E 0} \approx 0.7$ at present, and having set the present scale factor $a_{0}=1$. We use the redshift $z=-1+a_{0} / a$ as independent variable. In the upper graph we present the evolution of the dark energy and matter density parameters. In the middle graph we depict the evolution of the dark-energy equation of state. In the lower graph we present the evolution of the deceleration parameter $q$.

scenario of bi-scalar gravity can lead to a cosmological behavior in agreement with observations. We mention that we have not considered neither an explicit cosmological constant nor a potential that could play the role of a cosmological constant in particular limits, and therefore the late-time acceleration is a pure result of the novel, bi-scalar construction and the involved couplings between the two scalars.

We now proceed to the investigation of how the two-scalar coupling parameters $\beta$ and $\gamma$ in action $S_{1}$ affect the cosmological evolution, and in particular the dark-energy equation-of-state parameter $w_{D E}$. In Fig. 2 we depict the evolution of $w_{D E}$ for various values of $\beta$ and $\gamma$. As we can see, there are parameter regions for which $w_{D E}$ lies in the quintessence regime, however there are also parameter regions for which $w_{D E}$ exhibits 
the phantom-divide crossing in the recent cosmological past, acquiring a value below the cosmological-constant one at present. This is a significant advantage of the present biscalar scenario, since the phantom behavior is acquired although the whole construction is free of ghosts and the two scalar fields are not phantom ones.

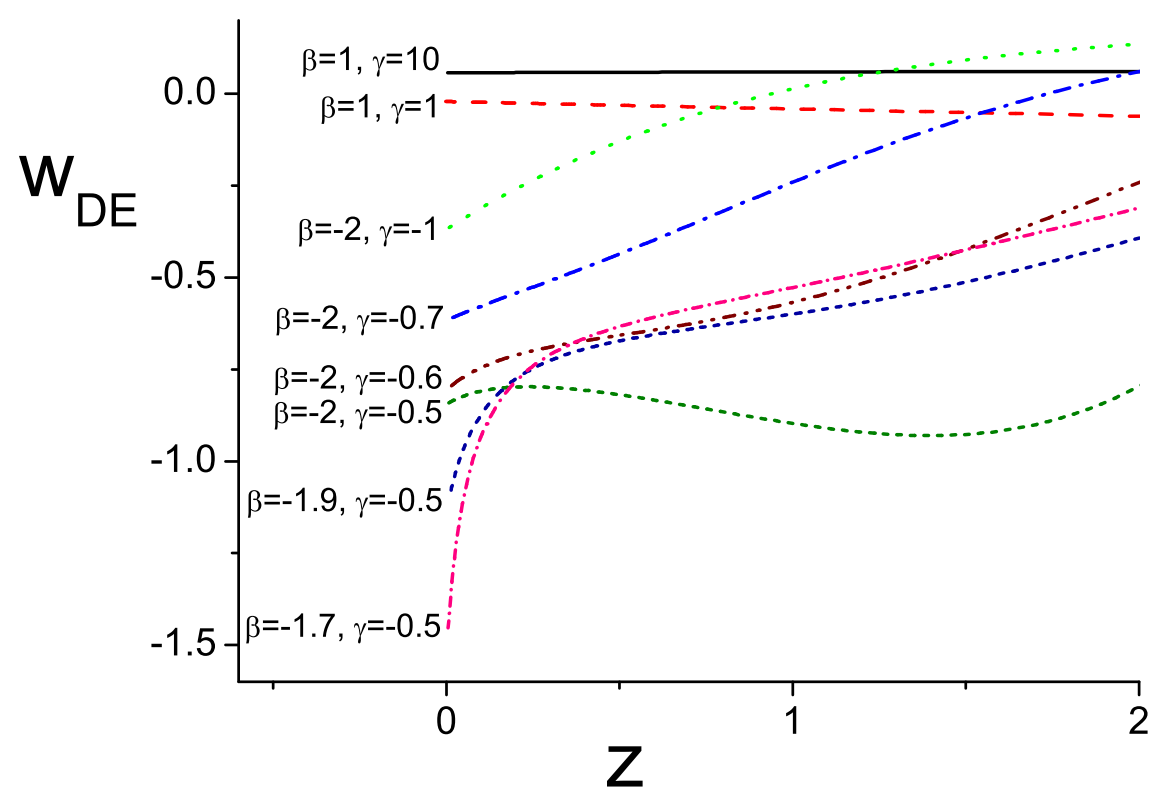

Figure 2. The evolution of the dark-energy equation-of-state parameter as a function of the redshift $z=-1+a_{0} / a$, for $\eta=1$, and for eight choices of the parameters $\beta$ and $\gamma$, in units where $8 \pi G=1$, having imposed $\Omega_{m 0} \approx 0.3, \Omega_{D E 0} \approx 0.7$ at present, and having set the present scale factor $a_{0}=1$.

\section{Conclusions}

In this work we investigated the cosmological applications of a recently proposed gravitational modification, corresponding to a bi-scalar theory constructed in a way to exhibit partial conformal invariance, which could become full conformal invariance in the absence of the usual Einstein-Hilbert term and introducing additionally either the Weyl derivative or properly rescaled fields $[35,50]$. Such a theory is constructed by considering the action of a non-minimally conformally coupled scalar field as a "seed" action, in which one adds a second scalar allowing for a nonminimal derivative coupling with the Einstein tensor and the energy-momentum tensor of the first conformally-coupled scalar field. In this way, the equation of motion of the second field can be written as a current conservation equation, with the current enclosing the metric field equations of the initial "seed" action.

Applying this bi-scalar modified gravity in a cosmological framework, we extracted the Friedmann equations as well as evolution equations of the two scalar fields, obtaining 
an effective dark-energy sector constituted from both scalars. Firstly, we extracted various analytical solutions in the absence of an explicit matter sector. Interestingly enough, for some specific solution subclasses we saw that we can obtain a universe dynamics corresponding to matter-era evolution, although we have not considered an explicit matter sector. This important feature results from the fact that for this parameter region the effective dark-energy equation-of-state parameter becomes zero, and thus the scalar fields give rise not to an effective-dark energy sector, but to an affective dark-matter one, i.e. to a form of "mimetic dark matter". Additionally, for different parameter regions we showed that we can obtain both an effective dark-matter sector, as well as an effective dark-radiation sector, and hence obtaining the thermal history of the universe, with an initial radiation era followed by the matter epoch. Finally, there are parameter regions that allow for an arbitrary scale-factor evolution, which can have important implications, since apart from late-time acceleration they can describe the complete thermal history of the universe, namely the sequence from inflation, to radiation, to matter and to dark-energy epochs, as well as alternatively evolutions such as the bouncing or cyclic ones.

In the case where an explicit matter sector is included, we evolved the full cosmological system numerically, focusing on various observables such as the matter and dark-energy density parameters, the deceleration parameter, and the effective dark energy equation of state. As we saw, the obtained cosmological evolution is in agreement with observations, with the matter era followed by the dark-energy epoch and the transition to the cosmic acceleration. Furthermore, for particular regions of the model parameters, the effective dark-energy equation-of-state parameter can pass through the cosmological constant value, resulting in the phantom regime at present. This feature reveals the capabilities of the (partially) conformally invariant bi-scalar theory, since the phantom behavior is acquired although the fields are canonical and the theory is ghost free. We stress here that the above behaviors are obtained without the presence of an explicit cosmological constant, or of a potential that could play the role of a cosmological constant in particular limits, i.e. they arise purely from the novel, bi-scalar construction and the involved couplings between the two fields.

In summary, (partially) conformally invariant bi-scalar theories have interesting cosmological implications in agreement with observations. Thus, it would be worthy to further investigate them, confronting them with observations using Type Ia Supernovae (SNIa), Baryon Acoustic Oscillations (BAO), and Cosmic Microwave Background (CMB) data, as well as analyzing the perturbations and constraining them with large-scale structure observations. Additionally, one could perform a detailed dynamical-system analysis, in order to by-pass the non-linearities and reveal the asymptotic cosmological behavior. These studies are left for future projects.

\section{Acknowledgments}

MT is supported by TUBITAK 2216 fellowship under the application number 1059B161500790. 


\section{References}

[1] A. Joyce, B. Jain, J. Khoury and M. Trodden, Beyond the Cosmological Standard Model, Phys. Rept. 568 (2015) 1, [arXiv:1407.0059].

[2] T. Clifton, P. G. Ferreira, A. Padilla and C. Skordis, Modified Gravity and Cosmology, Phys. Rept. 513 (2012) 1, [arXiv:1106.2476].

[3] E. J. Copeland, M. Sami and S. Tsujikawa, Dynamics of dark energy, Int. J. Mod. Phys. D 15, 1753 (2006), [arXiv:hep-th/0603057].

[4] Y. F. Cai, E. N. Saridakis, M. R. Setare and J. Q. Xia, Quintom Cosmology: Theoretical implications and observations, Phys. Rept. 493, 1 (2010), [arXiv:0909.2776].

[5] J. Martin, C. Ringeval and V. Vennin, Encyclopdia Inflationaris, Phys. Dark Univ. 5-6, 75 (2014), [arXiv: 1303.3787].

[6] M. Ostrogradsky, Memoire sur les quadratures definies, Memoires de l'Academie Imperiale des Science de Saint-Petersbourg, 4:385, 1850.

[7] R. P. Woodard, Avoiding dark energy with 1/r modifications of gravity, Lect. Notes Phys. 720 (2007) 403, [arXiv:astro-ph/0601672].

[8] R. P. Woodard, Ostrogradsky's theorem on Hamiltonian instability, Scholarpedia 10 (2015) 8, 32243, [arXiv: 1506.02210].

[9] Y. Fujii and K. Maeda, The scalar-tensor theory of gravitation, Cambridge University Press, (2003).

[10] C. Charmousis, E. J. Copeland, A. Padilla and P. M. Saffin, General second order scalar-tensor theory, self tuning, and the Fab Four, Phys. Rev. Lett. 108 (2012) 051101, [arXiv: 1106.2000].

[11] T. Kobayashi, M. Yamaguchi and J. Yokoyama, Generalized G-inflation: Inflation with the most general second-order field equations, Prog. Theor. Phys. 126 (2011) 511, [arXiv: 1105.5723].

[12] C. Deffayet, X. Gao, D. A. Steer and G. Zahariade, From k-essence to generalised Galileons, Phys. Rev. D 84 (2011) 064039, [arXiv:1103.3260].

[13] G. W. Horndeski, Second-order scalar-tensor field equations in a four-dimensional space, Int. J. Theor. Phys. 10 (1974) 363.

[14] J. Gleyzes, D. Langlois, F. Piazza and F. Vernizzi, Healthy theories beyond Horndeski, Phys. Rev. Lett. 114 (2015) 21, 211101, [arXiv:1404.6495].

[15] J. Gleyzes, D. Langlois, F. Piazza and F. Vernizzi, Exploring gravitational theories beyond Horndeski, JCAP 1502 (2015) 018, [arXiv:1408.1952].

[16] X. Gao, Unifying framework for scalar-tensor theories of gravity, Phys. Rev. D 90 (2014) 081501, [arXiv:1406.0822].

[17] M. Zumalacregui and J. Garc-Bellido, Transforming gravity: from derivative couplings to matter to second-order scalar-tensor theories beyond the Horndeski Lagrangian, Phys. Rev. D 89 (2014) 064046, [arXiv: 1308.4685].

[18] G. Domech, S. Mukohyama, R. Namba, A. Naruko, R. Saitou and Y. Watanabe, Derivative-dependent metric transformation and physical degrees of freedom, Phys. Rev. D 92 (2015) 8, 084027, [arXiv: 1507.05390]. 
[19] D. Langlois and K. Noui, Degenerate higher derivative theories beyond Horndeski: evading the Ostrogradski instability, [arXiv:1510.06930].

[20] C. Deffayet, G. Esposito-Farese and D. A. Steer, Counting the degrees of freedom of generalized Galileons, Phys. Rev. D 92 (2015) 084013, [arXiv:1506.01974].

[21] D. Langlois and K. Noui, Hamiltonian analysis of higher derivative scalar-tensor theories, [arXiv: 1512.06820].

[22] M. Crisostomi, M. Hull, K. Koyama and G. Tasinato, Horndeski: beyond, or not beyond?, [arXiv: 1601.04658 ].

[23] M. Crisostomi, K. Koyama and G. Tasinato, Extended Scalar-Tensor Theories of Gravity, [arXiv: 1602.03119].

[24] A. Nicolis, R. Rattazzi and E. Trincherini, The Galileon as a local modification of gravity, Phys. Rev. D 79 (2009) 064036. [arXiv:0811.2197].

[25] C. Deffayet, G. Esposito-Farese and A. Vikman, Covariant Galileon, Phys. Rev. D 79, 084003 (2009), [arXiv:0901.1314].

[26] C. Deffayet, S. Deser and G. Esposito-Farese, Generalized Galileons: All scalar models whose curved background extensions maintain second-order field equations and stress-tensors, Phys. Rev. D 80, 064015 (2009), [arXiv: 0906.1967].

[27] A. Padilla, P. M. Saffin and S. Y. Zhou, Bi-galileon theory I: Motivation and formulation, JHEP 1012 (2010) 031, [arXiv:1007.5424].

[28] A. Padilla and V. Sivanesan, Covariant multi-galileons and their generalisation, JHEP 1304 (2013) 032, [arXiv: 1210.4026].

[29] V. Sivanesan, Generalized multiple-scalar field theory in Minkowski space-time free of Ostrogradski ghosts, Phys. Rev. D 90 (2014) 10, 104006, [arXiv: 1307.8081].

[30] T. Kobayashi, N. Tanahashi and M. Yamaguchi, Multifield extension of $G$ inflation, Phys. Rev. D 88 (2013) 8, 083504, [arXiv: 1308.4798].

[31] S. Ohashi, N. Tanahashi, T. Kobayashi and M. Yamaguchi, The most general second-order field equations of bi-scalar-tensor theory in four dimensions, JHEP 1507 (2015) 008, [arXiv: 1505.06029].

[32] A. Naruko, D. Yoshida and S. Mukohyama, Gravitational scalar-tensor theory, [arXiv: 1512.06977].

[33] E. N. Saridakis and M. Tsoukalas, Cosmology in new gravitational scalar-tensor theories, [arXiv: 1601.06734 ].

[34] A. Padilla, P. M. Saffin and S. Y. Zhou, Bi-galileon theory II: Phenomenology, JHEP 1101, 099 (2011), [arXiv: 1008.3312].

[35] A. Padilla, D. Stefanyszyn and M. Tsoukalas, Generalised Scale Invariant Theories, Phys. Rev. D 89 (2014) 6, 065009, [arXiv:1312.0975].

[36] G. 'T Hooft, Local conformal symmetry: The missing symmetry component for space and time, Int. J. Mod. Phys. D 24 (2015) 12, 1543001.

[37] J. L. Sievers et al. [Atacama Cosmology Telescope Collaboration], The Atacama Cosmology Telescope: Cosmological parameters from three seasons of data, JCAP 1310 (2013) 060, [arXiv: 1301.0824]. 
[38] P. A. R. Ade et al. [Planck Collaboration], Planck 2015 results. XIII. Cosmological parameters, [arXiv: 1502.01589].

[39] I. Bars, S. -H. Chen and N. Turok, Geodesically Complete Analytic Solutions for a Cyclic Universe, Phys. Rev. D 84 (2011) 083513, [arXiv:1105.3606].

[40] I. Bars, S. -H. Chen, P. J. Steinhardt and N. Turok, Antigravity and the Big Crunch/Big Bang Transition, Phys. Lett. B 715 (2012) 278, [arXiv:1112.2470].

[41] I. Bars, S. -H. Chen, P. J. Steinhardt and N. Turok, Complete Set of Homogeneous Isotropic Analytic Solutions in Scalar-Tensor Cosmology with Radiation and Curvature, Phys. Rev. D 86 (2012) 083542, [arXiv: 1207.1940].

[42] I. Bars, Traversing Cosmological Singularities, Complete Journeys Through Spacetime Including Antigravity, [arXiv:1209.1068].

[43] I. Bars, P. Steinhardt and N. Turok, Local Conformal Symmetry in Physics and Cosmology, [arXiv: 1307.1848].

[44] J. J. M. Carrasco, W. Chemissany and R. Kallosh, Journeys Through Antigravity?, [arXiv: 1311.3671].

[45] R. Kallosh and A. Linde, Universality Class in Conformal Inflation, JCAP 1307 (2013) 002, [arXiv: 1306.5220].

[46] G. 't Hooft, Probing the small distance structure of canonical quantum gravity using the conformal group, [arXiv: 1009.0669].

[47] P. D. Mannheim and D. Kazanas, Astrophys. J. 342, 635 (1989).

[48] E. E. Flanagan, Fourth order Weyl gravity, Phys. Rev. D 74, 023002 (2006), [arXiv: astro-ph/0605504].

[49] J. Maldacena, Einstein Gravity from Conformal Gravity, [arXiv:1105.5632].

[50] C. Charmousis, T. Kolyvaris, E. Papantonopoulos and M. Tsoukalas, Black Holes in Bi-scalar Extensions of Horndeski Theories, JHEP 1407 (2014) 085, [arXiv:1404.1024].

[51] N. M. Bocharova, K. A. Bronnikov and V. N. Melnikov, An exact solution of the system of einstein equations and mass-free scalar field, Vestn. Mosk. Univ. Fiz. Astro. 6 (1970) 706.

[52] J. D. Bekenstein, Exact solutions of Einstein conformal scalar equations, Annals Phys. 82, 535 (1974).

[53] C. Martinez, R. Troncoso and J. Zanelli, Exact black hole solution with a minimally coupled scalar field, Phys. Rev. D 70, 084035 (2004), [arXiv:hep-th/0406111].

[54] C. Martinez, J. P. Staforelli and R. Troncoso, Topological black holes dressed with a conformally coupled scalar field and electric charge, Phys. Rev. D 74, 044028 (2006), [arXiv:hep-th/0512022].

[55] C. Martinez and R. Troncoso, Electrically charged black hole with scalar hair, Phys. Rev. D 74, 064007 (2006), [arXiv:hep-th/0606130].

[56] C. Martinez, R. Troncoso and J. Zanelli, De Sitter black hole with a conformally coupled scalar field in four-dimensions, Phys. Rev. D 67 (2003) 024008, [arXiv:hep-th/0205319].

[57] E. N. Saridakis and S. V. Sushkov, Quintessence and phantom cosmology with non-minimal derivative coupling, Phys. Rev. D 81, 083510 (2010), [arXiv: 1002.3478].

[58] S. Deser, Gravitation, Scale Invariance and Cosmology and Universal Self Coupling in Gauge 
Theories, Ann. Phys. 59248.

[59] H. Ogawa, T. Kobayashi and T. Suyama, Instability of hairy black holes in shift-symmetric Horndeski theories, [arXiv: 1510.07400].

[60] A. H. Chamseddine and V. Mukhanov, Mimetic Dark Matter, JHEP 1311, 135 (2013), [arXiv: 1308.5410].

[61] G. Leon and E. N. Saridakis, Dynamical behavior in mimetic $F(R)$ gravity, JCAP 1504, no. 04, 031 (2015), [arXiv: 1501.00488].

[62] F. Bernardeau, S. Colombi, E. Gaztanaga and R. Scoccimarro, Large scale structure of the universe and cosmological perturbation theory, Phys. Rept. 367, 1 (2002), [arXiv: astro-ph/0112551].

[63] M. Novello and S. E. P. Bergliaffa, Bouncing Cosmologies, Phys. Rept. 463, 127 (2008), [arXiv:0802.1634].

[64] S. Mukohyama, Brane world solutions, standard cosmology, and dark radiation, Phys. Lett. B 473, 241 (2000), [arXiv:hep-th/9911165].

[65] E. N. Saridakis, Horava-Lifshitz Dark Energy, Eur. Phys. J. C 67, 229 (2010), [arXiv: 0905.3532].

[66] P. J. E. Peebles, Principles of physical cosmology, Princeton, USA: Univ. Pr. (1993). 